Revista Brasileira de Agricultura Irrigada v.13, nº.4, p. 3533 - 3539, 2019

ISSN 1982-7679 (On-line)

Fortaleza, CE, INOVAGRI - http://www.inovagri.org.br

DOI: $10.7127 /$ rbai.v13n4001077

Protocolo 1077.19 - 15/03/2019 Aprovado em 05/07/52019

\title{
DESEMPENHO PRODUTIVO DE CULTIVARES DE MELANCIA EM DUAS ÉPOCAS DE CULTIVO NO SUBMÉDIO DO VALE DO SÃO FRANCISCO
}

\author{
Geraldo Milanez de Resende ${ }^{1 *}$, Jony Eshi Yuri ${ }^{1}$
}

\begin{abstract}
RESUMO
A escolha criteriosa da cultivar com a definição dos melhores materiais que se adaptam às condições locais de cultivo é decisiva para o sucesso do cultivo da melancia, na lucratividade da cultura bem como na competitividade do produtor. $\mathrm{O}$ objetivo do presente trabalho foi avaliar a produtividade de cultivares de melancia sob cultivo em temperaturas amenas (inverno) e mais elevadas (verão) nas condições do Submédio do Vale do São Francisco no período de maio a julho e agosto a outubro de 2017, em Petrolina-PE. Os experimentos foram conduzidos no delineamento experimental de blocos ao acaso com quatorze cultivares no inverno e doze cultivares no verão, com três repetições. No cultivo sob temperaturas amenas (inverno) maiores produtividades comerciais foram observadas para as cultivares Frevo (45,7 $\left.\mathrm{t} \mathrm{ha}^{-1}\right)$, Red Heaven $\left(48,2 \mathrm{t} \mathrm{ha}^{-1}\right)$, Explorer $\left(48,5 \mathrm{t} \mathrm{ha}^{-1}\right)$ e Ranger (49,9 $\mathrm{tha}$ ${ }^{1}$ ), sendo que as cultivares menos produtivas obtiveram índices abaixo de $37,0 \mathrm{t} \mathrm{ha}^{-1}$. Entre essas, a massa fresca de fruto comercial variou entre $8,7 \mathrm{~kg}^{\text {fruto }}{ }^{-1}$ (cultivar Red Heaven) e $10,2 \mathrm{~kg}$ fruto (Cultivar Explorer). Para o cultivo sob temperaturas mais elevadas, as maiores produtividades comerciais de frutos foram apresentadas pelas cultivares Opara $F_{1}\left(51,6 \mathrm{tha}^{-1}\right)$, Ranger $\left(53,8 \mathrm{tha}^{-1}\right)$, Combat (55,2 $\left.\mathrm{t} \mathrm{ha}^{-1}\right)$, Explorer (55,6 $\left.\mathrm{tha}^{-1}\right)$, Conquista $\left(56,0 \mathrm{t} \mathrm{ha}^{-1}\right)$, Frevo $\left(56,7 \mathrm{t} \mathrm{ha}^{-1}\right)$ e Red Heaven $\left(57,2 \mathrm{t} \mathrm{ha}^{-1}\right)$. Entre as cultivares mais produtivas verificou-se que a massa fresca do fruto oscilou entre $8,3 \mathrm{~kg}_{\text {fruto }}^{-1}$ (cultivar Opara $\mathrm{F}_{1}$ ) e 11,0 $\mathrm{kg}_{\text {fruto }}{ }^{-1}$ (cultivar Red Heaven).
\end{abstract}

Palavras-chave: Citrullus lanatus, competição, época de plantio, produtividade.

\section{AGRONOMICAL PERFORMANCE OF WATERMELON CULTIVARS IN TWO GROWING SEASONS AT SUB-MIDDLE SÃO FRANCISCO VALLEY}

\begin{abstract}
The careful selection of the cultivar with the definition of the best materials that adapt to the local conditions of cultivation is decisive for the success of the watermelon cultivation, the profitability of the crop as well as the competitiveness of the farmer. The objective of the present study was to evaluate the yield of watermelon cultivars under cultivation at mild (winter) and higher (summer) temperatures under the conditions of the Submiddle of São Francisco Valley, in the period from
\end{abstract}

\footnotetext{
1 * Autor para correspondência Eng. Agrônomo, D.Sc., Embrapa - Centro de Pesquisa Agropecuária do Trópico Semiárido. E-mails: geraldo.milanez@embrapa.br; jony.yuri@embrapa.br
} 
April to July and August to October 2017, in Petrolina-PE, Brazil. The experiments were conducted in a randomized complete block design with fourteen cultivars in winter and twelve cultivars in the summer, with three replications. The cultivars Frevo $\left(45.7 \mathrm{t} \mathrm{ha}^{-1}\right)$, Red Heaven $\left(48.2 \mathrm{t} \mathrm{ha}^{-1}\right)$, Explorer (48.5 $\left.\mathrm{t} \mathrm{ha}^{-1}\right)$ and Ranger (49.9 $\mathrm{t} \mathrm{ha}^{-1}$ ) were the most productive, with less productive cultivars having indices below $37.0 \mathrm{t} \mathrm{ha}^{-1}$. Among these, fresh commercial fruit mass ranged from $8.7 \mathrm{~kg}_{\text {fruit }}{ }^{-1}$ (Red Heaven cultivar) to $10.2 \mathrm{~kg}$ fruit $^{-1}$ (Cultivar Explorer). For the cultivation under higher temperatures the highest commercial fruit yields were presented by the cultivars Opara F1 (51.6 $\left.\mathrm{t} \mathrm{ha}^{-1}\right)$, Ranger $\left(53.8 \mathrm{t} \mathrm{ha}^{-1}\right)$, Combat $\left(55.2 \mathrm{t} \mathrm{ha}^{-1}\right)\left(56.7 \mathrm{t} \mathrm{ha}^{-1}\right)$, Red $\left(57.2 \mathrm{t} \mathrm{ha}^{-1}\right)$ and Frevo $\left(56.7 \mathrm{t} \mathrm{ha}^{-1}\right)$. It was verified that the fresh mass of the fruit varied between $8.3 \mathrm{~kg}^{-1}$ fruit $^{-1}$ (cultivar Opara F1) and $11.0 \mathrm{~kg}$ fruit $^{-1}$ (cultivar Red Heaven).

Keywords: Citrullus lanatus, competition, planting date, yield.

\section{INTRODUÇÃO}

A melancia (Citrullus lanatus (Thunb.) Matsun. \& Nakai) tem grande importância econômica e social. Segundo a FAO (2018) em 2016 a produção mundial atingiu 117,02 milhões de toneladas com produtividade de 33,6 $\mathrm{t} \mathrm{ha}^{-1}$, tendo o Brasil produzido 2,09 milhões de toneladas com produtividade de 23,11 $\mathrm{t} \mathrm{ha}{ }^{-1}$. O Nordeste respondeu por $31,06 \%$ da produção nacional alcançando produtividade $18,56 \mathrm{t} \mathrm{ha}^{-1}$, sendo a Bahia o maior estado produtor com $237.532 \mathrm{t}$ e produtividade de 16,72 $\mathrm{t} \mathrm{ha}^{-1}$. Em Pernambuco foram cultivados 2.629 hectares com produtividade de 19,25 $\mathrm{t} \mathrm{ha}^{-1}$ (IBGE, 2018).

A família Cucurbitaceae se caracteriza por adaptar melhor às zonas quentes $\mathrm{e}$ semiáridas com maior luminosidade $\mathrm{e}$ temperaturas médias entre $18{ }^{\circ} \mathrm{C}$ a $30{ }^{\circ} \mathrm{C}$, não suportando temperaturas menores que $10{ }^{\circ} \mathrm{C}$. Dentre as diferentes espécies que compõem as cucurbitáceas, a melancia é a que menos tolera baixas temperaturas, sobretudo no período de desenvolvimento da cultura (germinação e emergência), sendo tipicamente uma cultura de clima quente. A temperatura média do ar mais adequada para seu crescimento e desenvolvimento deve estar em torno de $25{ }^{\circ} \mathrm{C}$ (RESENDE et al., 2010a).

O cultivo da melancia no Nordeste se realiza sob condições de sequeiro em consórcio com outras culturas de subsistência de forma tradicional por pequenos agricultores no período de ocorrência de chuvas, ou seja, de dezembro a março. No âmbito mais comercial é realizado em condições de irrigação nos Perímetros do Vale do São Francisco e são cultivados durante todo o ano, com maior concentração de plantios entre os meses de agosto a outubro (RESENDE et al., 2010b).

É uma planta bastante influenciada por condições ambientais, sobretudo temperatura. $\mathrm{O}$ inverno (temperaturas mais amenas) ocorre de abril a setembro e o verão (temperaturas mais elevadas) de outubro a março nas condições do Submédio do Vale do São Francisco. Nesse contexto é de se esperar que a melancia deva se desenvolver e produzir melhor em períodos de temperaturas mais elevadas (verão) comparativamente a época de inverno (temperaturas mais amenas). A temperatura média do ar varia de 24,0 a 28,0 ${ }^{\circ} \mathrm{C}$, com as temperaturas máxima e mínima oscilando entre 29,6 a $34,0{ }^{\circ} \mathrm{C}$ e de 18,2 a 22,1 ${ }^{\circ} \mathrm{C}$, respectivamente, para as condições de inverno e verão (TEIXEIRA, 2010).

Em geral existe no mercado grande disponibilidade de cultivares com diferentes formatos de fruto, coloração externa, tolerância a doenças e ao transporte, tamanho padrão e características sensoriais, especialmente, sabor e aparência. Além desse contexto, as cultivares variam também pela sua capacidade de resposta em termos de produtividade às diferentes condições de cultivo em que são submetidas (FERREIRA et al., 2003).

Estudos realizados por Carmo et al. (2015) sob condições de temperaturas mais 
elevadas (dezembro a fevereiro) demonstraram ser as cultivares Crimson Sweet e Explorer as mais produtivas $(63,2$ e $60,1 \mathrm{t}$ $\left.\mathrm{ha}^{-1}\right)$, assim como as que apresentaram maior número de frutos por hectare com $8.103 \mathrm{e}$ 7.160 frutos, respectivamente. Com relação à massa fresca do fruto observaram variações entre 7,1 a $9,0 \mathrm{~kg}$ fruto entre as cultivares avaliadas, $7,8 \mathrm{~kg}$ fruto $^{-1}$ para a cultivar Crimson Sweet e 8,4 kg fruto ${ }^{-1}$ para a cultivar Explorer. Nas mesmas condições, Silva et al. (2017) verificaram que a cultivar Crimson Select Plus foi a mais produtiva $\left(67.8 \mathrm{t} \mathrm{ha}^{-1}\right)$, seguida pelas cultivares Santa Amélia (61,5 t $\mathrm{ha}^{-1}$ ) e Explore $\left(60,2 \mathrm{t} \mathrm{ha}^{-1}\right)$ com variações no número de frutos por hectare e massa fresca de fruto entre 5.375 a 6.425 e 9,68 a $11,31 \mathrm{~kg}$ fruto $^{-1}$, respectivamente.

O presente trabalho objetivou avaliar diferentes cultivares de melancia em duas épocas de plantio nas condições do Submédio do Vale do São Francisco.

\section{MATERIAIS E MÉTODOS}

Os experimentos foram conduzidos sob condições de temperaturas amenas, no período de maio a julho, e agosto a outubro de 2017, sob temperaturas mais elevadas, no Campo Experimental de Bebedouro, Petrolina-PE ( $9^{\circ} 9^{\prime} \mathrm{S}, 40^{\circ} 29^{\prime \prime} \mathrm{W}, 365,5 \mathrm{~m}$ de altitude). O solo foi classificado como Latossolo Vermelho Amarelo Distróferrico (SANTOS et al., 2018). A precipitação pluviométrica acumulada, temperaturas máxima, mínima e média e umidade relativa do ar nos períodos de execução do experimento no campo encontram-se na Tabela 1.

$\mathrm{O}$ delineamento experimental utilizado foi de blocos ao acaso com três repetições, sendo quatorze cultivares no plantio sob temperaturas mais amenas de inverno (Combat, Conquista, Crimson Sweet, Electra, Explorer, Frevo, Maracatú, Olímpia, Omaru Yamato, Opara, Opara $F_{1}$, Ranger, Red Heaven e Talisman) e doze cultivares sob temperaturas mais elevadas de verão (Combat, Conquista, Crimson Sweet, Explorer, Frevo, Maracatú, Olímpia Omaru Yamato, Opara, Opara $F_{1}$, Ranger e Red Heaven).

A semeadura foi realizada em 01/05/2017, na primeira época (inverno) e em 10/08/2017 na segunda época (verão) em viveiro, com transplantios aos 12 e 10 dias após a semeadura (início da emergência da primeira folha definitiva), respectivamente. Foram utilizadas bandejas de isopor contendo 200 células, preenchidas com substrato comercial "Plantmax HT". O preparo do solo, feito da mesma forma nas duas épocas, e constou de aração, gradagem e sulcamento.

Tabela 1. Valores mensais de precipitação pluviométrica acumulada, temperaturas máxima, mínima e média e umidade relativa do ar média durante a execução dos experimentos em campo entre maio a julho (inverno) e agosto a outubro (verão). Embrapa Semiárido. Petrolina - PE, 2017.

\begin{tabular}{cccccc}
\hline \multirow{2}{*}{ Meses } & $\begin{array}{c}\text { Precipitação } \\
(\mathrm{mm})\end{array}$ & \multicolumn{3}{c}{ Temperatura $\left({ }^{\circ} \mathrm{C}\right)$} & Umidade relativa (\%) \\
\cline { 3 - 5 } & 26 & Máxima & Mínima & Média & 65,1 \\
Maio & 9 & 32,7 & 21,9 & 27,0 & 74,1 \\
Junho & 5 & 30,4 & 18,8 & 24,2 & 72,5 \\
Julho & - & 36,8 & 18,0 & 22,5 & 68,1 \\
\hline Média & 0 & 31,8 & 19,6 & 24,6 & 64,4 \\
\hline Agosto & 12 & 30,1 & 19,2 & 25,1 & 68,7 \\
Setembro & 0 & 34,8 & 21,6 & 24,1 & 65,4 \\
Outubro & - & 32,2 & 19,8 & 27,8 & 66,1 \\
\hline Média & - & &
\end{tabular}

As parcelas experimentais constituíramse de duas linhas de $4,2 \mathrm{~m}$ de comprimento espaçadas de $3,0 \mathrm{~m}\left(25,2 \mathrm{~m}^{2}\right)$, sendo entre plantas de $0,60 \mathrm{~m}$. As duas linhas centrais formaram a área útil $\left(18,0 \mathrm{~m}^{2}\right)$, retirando-se uma planta em cada extremidade. Deixou-se entre blocos uma distancia de $1,2 \mathrm{~m}$, sendo as bordaduras externas aos experimentos.

As adubações com base na análise do solo constaram da aplicação de 500,0 $\mathrm{kg} \mathrm{ha}^{-1}$ 
do formulado NPK 06-24-12 no plantio. Em adubação de cobertura, foram adicionados 90,0 $\mathrm{kg} \mathrm{ha}^{-1}$ de $\mathrm{N}, 90,0 \mathrm{~kg} \mathrm{ha}^{-1}$ de $\mathrm{K}_{2} \mathrm{O}, 40,0$ $\mathrm{kg} \mathrm{ha}{ }^{-1}$ de $\mathrm{Ca}$ e 10,0 $\mathrm{kg} \mathrm{ha}^{-1}$ de $\mathrm{Mg}$, via fertirrigação, três vezes por semana, ao longo do ciclo, até 7 antes da colheita.

A cultura foi mantida no limpo através de capinas manuais, quando necessárias. As irrigações foram feitas através do método de gotejamento, com turno diário e lâminas de água em torno de 7 - $8 \mathrm{~mm}$ (inverno) e 9 - 11 $\mathrm{mm}$ (verão), calculada em função da evaporação do tanque classe A. Foram realizados também os tratos fitossanitários comuns à cultura.

As colheitas foram realizadas aos 74 dias e 67 dias após o transplantio, respectivamente para o cultivo de primeiro e segundo plantio, quando os frutos apresentaram-se completamente desenvolvidos, sendo avaliadas a produtividade total e comercial $\left(\mathrm{t} \mathrm{ha}^{-1}\right)$, massa fresca do fruto comercial $\left(\mathrm{kg}_{\text {fruto }}{ }^{-1}\right)$ e número de frutos por planta e por hectare. Os dados foram submetidos ao teste de normalidade de Shapiro-Wilk e, quando verificada a normalidade $(\mathrm{p}>0,05)$, que indicaram a não necessidade de transformação, desse modo se aplicou a análise de variância (ANOVA), e as médias comparadas pelo teste de Scott-Knott, a 5\% de significância. Todas as análises foram realizadas empregando-se o programa SISVAR (FERREIRA, 2014).

\section{RESULTADOS E DISCUSSÃO}

Os resultados evidenciaram efeitos significativos das cultivares em função das duas épocas de plantio para produtividade total e comercial e massa fresca de fruto, sem diferenças significativas para número de frutos por planta e por hectare (Tabelas 2 e 3 ).

No cultivo sob temperaturas amenas (inverno) no Submédio do Vale do São Francisco (com médias de temperaturas mínimas de $19,6{ }^{\circ} \mathrm{C}$ e máximas de $30,0{ }^{\circ} \mathrm{C}$ ) (Tabela 1), as cultivares de melancia Olímpia, Ranger, Frevo, Explorer e Red Heaven alcançaram as maiores produtividades totais de frutos, com valores acima de 46,0 $\mathrm{t} \mathrm{ha}^{-1}$ (Tabela 2).

Tabela 2. Produtividade total e comercial, massa fresca e número de frutos comerciais de cultivares de melancia sob condições de temperaturas mais amenas (inverno) no Submédio do Vale do São Francisco. Petrolina, 2017.

\begin{tabular}{cccccc}
\hline \multirow{2}{*}{ Cultivares } & \multicolumn{2}{c}{ Produtividade $\left(\mathrm{t} \mathrm{ha}^{-1}\right)$} & Massa fresca do & \multicolumn{2}{c}{ Número de frutos } \\
\cline { 2 - 3 } Oruto $(\mathrm{g})$ & Total & Comercial & & Planta & Hectare \\
\hline Omaru Yamato & $32,9 \mathrm{c}$ & $29,2 \mathrm{c}$ & $6,3 \mathrm{~b}$ & $0,85 \mathrm{a}$ & $4.722 \mathrm{a}$ \\
Conquista & $33,3 \mathrm{c}$ & $31,0 \mathrm{c}$ & $7,4 \mathrm{~b}$ & $0,76 \mathrm{a}$ & $4.197 \mathrm{a}$ \\
Opara & $35,2 \mathrm{c}$ & $30,1 \mathrm{c}$ & $6,6 \mathrm{~b}$ & $0,82 \mathrm{a}$ & $4.567 \mathrm{a}$ \\
Crimson Sweet & $35,5 \mathrm{c}$ & $32,2 \mathrm{c}$ & $7,3 \mathrm{~b}$ & $0,79 \mathrm{a}$ & $4.413 \mathrm{a}$ \\
Combat & $36,4 \mathrm{c}$ & $33,1 \mathrm{c}$ & $6,9 \mathrm{~b}$ & $0,87 \mathrm{a}$ & $4.814 \mathrm{a}$ \\
Talisman & $38,4 \mathrm{~b}$ & $36,6 \mathrm{c}$ & $7,8 \mathrm{~b}$ & $0,82 \mathrm{a}$ & $4.567 \mathrm{a}$ \\
Electra & $39,3 \mathrm{~b}$ & $31,7 \mathrm{c}$ & $7,6 \mathrm{~b}$ & $0,75 \mathrm{a}$ & $4.166 \mathrm{a}$ \\
Maracatún & $41,8 \mathrm{~b}$ & $39,5 \mathrm{~b}$ & $9,5 \mathrm{a}$ & $0,75 \mathrm{a}$ & $4.166 \mathrm{a}$ \\
Opara F $\mathrm{F}_{1}$ & $41,9 \mathrm{~b}$ & $40,5 \mathrm{~b}$ & $8,4 \mathrm{a}$ & $0,88 \mathrm{a}$ & $4.876 \mathrm{a}$ \\
Olímpia & $46,9 \mathrm{a}$ & $41,6 \mathrm{~b}$ & $8,8 \mathrm{a}$ & $0,86 \mathrm{a}$ & $4.783 \mathrm{a}$ \\
Ranger & $50,8 \mathrm{a}$ & $49,9 \mathrm{a}$ & $9,7 \mathrm{a}$ & $0,93 \mathrm{a}$ & $5.185 \mathrm{a}$ \\
Frevo & $51,0 \mathrm{a}$ & $45,7 \mathrm{a}$ & $9,7 \mathrm{a}$ & $0,86 \mathrm{a}$ & $4.783 \mathrm{a}$ \\
Explorer & $51,3 \mathrm{a}$ & $48,5 \mathrm{a}$ & $10,2 \mathrm{a}$ & $0,87 \mathrm{a}$ & $4.814 \mathrm{a}$ \\
Red Heaven & $55,6 \mathrm{a}$ & $48,2 \mathrm{a}$ & $8,7 \mathrm{a}$ & $1,00 \mathrm{a}$ & $5.555 \mathrm{a}$ \\
\hline CV. $(\%)$ & 8,7 & 7,0 & 10,7 & 11,6 & 11,7 \\
\hline
\end{tabular}

*Médias seguidas pela mesma letra, na coluna, não diferem entre si, pelo teste de Scott-Knott a 5\% de probabilidade. 
Com relação a produtividade comercial (Tabela 2) as cultivares Frevo (45,7 $\left.\mathrm{t} \mathrm{ha}^{-1}\right)$, Red Heaven (48,2 $\left.\mathrm{t} \mathrm{ha}^{-1}\right)$, Explorer (48,5 $\mathrm{t} \mathrm{ha}^{-1}$ ) e Ranger $\left(49,9 \mathrm{t} \mathrm{ha}^{-1}\right)$ apresentaram-se como as mais produtivas, sendo que as cultivares menos produtivas obtiveram indices abaixo de $37,0 \mathrm{t} \mathrm{ha}$. Resultados similares foram relatados por Oliveira et al. (2015) que observaram menor produtividade média $(43,4$ $\mathrm{t} \mathrm{ha}^{-1}$ ) no plantio sob condições de temperaturas mais amenas (junho) quando comparados ao de agosto, sob condições de temperaturas mais elevadas $\left(51,8 \mathrm{tha}^{-1}\right)$.

A escolha criteriosa da cultivar com a definição dos melhores materiais que se adaptam às condições locais de cultivo é decisiva para o sucesso do sistema de cultivo adotado, na lucratividade da cultura, como na competitividade do agricultor.

Entre as cultivares mais produtivas verificou-se massa fresca de fruto comercial com variações entre $8,7 \mathrm{~kg}$ fruto $^{-1}$ (cultivar Red Heaven) e 10,2 kg fruto $^{-1}$ (Cultivar Explorer) (Tabela 2). Estes resultados corroboram o relato de (CARDOSO; ISAAC, 2010) de que os frutos comerciáveis são de peso variável $(5,5 \mathrm{~kg}$ a $12,0 \mathrm{~kg})$, e no cômputo geral como peso médio entre $7,0 \mathrm{~kg}$ a $8,0 \mathrm{~kg}$. Em condições semelhantes de temperatura Oliveira et al. (2015) observaram valores inferiores, com oscilações entre 6,2 e $7,7 \mathrm{~kg}$ fruto $^{-1}$. O maior número de frutos por planta foram alcançados para as cultivares Red Heaven (1,0 frutos planta $\left.{ }^{-1}\right)$ e Ranger $(0,93$ frutos planta $\left.{ }^{-1}\right)$, com também o maior número de frutos comerciais por hectare com 5.185 ('Ranger') e 5.555 frutos para a 'Red Heaven' (Tabela 2), no entanto, sem diferirem das demais cultivares. Nesse quisito, Oliveira et al. (2015) verificaram valores bem superiores que variaram de 1,4 frutos planta $^{-1}$ (cultivar Denver) a 1,6 frutos planta ${ }^{-1}$ (Cultivar Crimson Sweet).

Para o cultivo sob temperaturas mais elevadas (com médias de temperaturas mínimas de $19,8^{\circ} \mathrm{C}$ e máximas de $32,2{ }^{\circ} \mathrm{C}$ ) (Tabela 1), as cultivares de melancia Opara $\mathrm{F}_{1}$, Ranger, Conquista, Combat, Frevo, Explorer e
Red Heaven alcançaram as maiores produtividades totais de frutos com valores acima de 53,0 $\mathrm{t}$ ha ${ }^{-1}$. Com relação a produtividade comercial as cultivares Opara $\mathrm{F}_{1}$ (51,6 $\left.\mathrm{t} \mathrm{ha}^{-1}\right)$, Ranger $\left(53,8 \mathrm{t} \mathrm{ha}^{-1}\right)$, Combat $\left(55,2 \mathrm{t} \mathrm{ha}^{-1}\right)$, Explorer $\left(55,6 \mathrm{t} \mathrm{ha}^{-1}\right)$, Conquista $\left(56,0 \mathrm{t} \mathrm{ha}^{-1}\right)$, Frevo $\left(56,7 \mathrm{t} \mathrm{ha}^{-1}\right)$ e Red Heaven $\left(57,2 \mathrm{t} \mathrm{ha}^{-1}\right)$ obtiveram as produtividades mais expressivas (Tabela 3). Carmo et al. (2015) sob condições de temperaturas mais elevadas (dezembro) observaram produtividades comerciais superiores para as cultivares Crimson Sweet e Explorer com 63,2 e 60,1 t $\mathrm{ha}^{-1}$, respectivamente. No mesmo período Silva et al. (2017) verificaram produtividades oscilando entre 40,8 e $67,8 \mathrm{t} \mathrm{ha}^{-1}$. Enquanto Oliveira et al. (2015) informaram valores médios similares em plantio de agosto $(51,8 \mathrm{t}$ $\mathrm{ha}^{-1}$ ). 
Tabela 3. Produtividade total e comercial, massa fresca e número de frutos comerciais de cultivares de melancia sob condições de temperaturas mais elevadas (verão) no Submédio do Vale do São Francisco. Petrolina, 2017.

\begin{tabular}{|c|c|c|c|c|c|}
\hline \multirow{2}{*}{ Cultivares } & \multicolumn{2}{|c|}{ Produtividade $\left(\mathrm{t} \mathrm{ha}^{-1}\right)$} & \multirow{2}{*}{$\begin{array}{l}\text { Massa fresca do } \\
\text { fruto }(\mathrm{g})\end{array}$} & \multicolumn{2}{|c|}{ Número de frutos } \\
\hline & Total & Comercial & & Planta & Hectare \\
\hline Omaru Yamato & $47,5 \mathrm{~b}$ & $45,1 \mathrm{~b}$ & $8,2 \mathrm{~b}$ & $1,00 \mathrm{a}$ & $5.555 \mathrm{a}$ \\
\hline Conquista & 56,0 a & 56,0 a & $8,6 \mathrm{~b}$ & $1,18 \mathrm{a}$ & $6.543 \mathrm{a}$ \\
\hline Opara & $45,1 \mathrm{~b}$ & $44,2 \mathrm{~b}$ & $7,2 \mathrm{~b}$ & $1,11 \mathrm{a}$ & $6.172 \mathrm{a}$ \\
\hline Crimson Sweet & $46,2 \mathrm{~b}$ & $46,2 \mathrm{~b}$ & $7,9 \mathrm{~b}$ & $1,05 \mathrm{a}$ & $5.863 \mathrm{a}$ \\
\hline Combat & 55,9 a & $55,2 \mathrm{a}$ & $10,5 \mathrm{a}$ & $0,96 \mathrm{a}$ & $5.308 \mathrm{a}$ \\
\hline Maracatu & $46,7 \mathrm{~b}$ & $46,7 \mathrm{~b}$ & $7,7 \mathrm{~b}$ & $1,11 \mathrm{a}$ & $6.172 \mathrm{a}$ \\
\hline Opara $F_{1}$ & $53,4 \mathrm{a}$ & $51,6 \mathrm{a}$ & $8,3 \mathrm{~b}$ & $1,12 \mathrm{a}$ & $6.234 \mathrm{a}$ \\
\hline Ranger & $53,8 \mathrm{a}$ & $53,8 \mathrm{a}$ & $9,7 \mathrm{a}$ & $1,01 \mathrm{a}$ & $5.617 \mathrm{a}$ \\
\hline Frevo & $56,7 \mathrm{a}$ & $56,7 \mathrm{a}$ & $9,2 \mathrm{a}$ & $1,11 \mathrm{a}$ & $6.172 \mathrm{a}$ \\
\hline Explorer & $56,6 \mathrm{a}$ & $55,6 \mathrm{a}$ & $9,1 \mathrm{a}$ & $1,11 \mathrm{a}$ & $6.172 \mathrm{a}$ \\
\hline Red Heaven & $57,8 \mathrm{a}$ & $57,2 \mathrm{a}$ & $11,0 \mathrm{a}$ & $0,94 \mathrm{a}$ & $5.246 \mathrm{a}$ \\
\hline CV. (\%) & 10,1 & 9,9 & 11,7 & 13,3 & 13,2 \\
\hline
\end{tabular}

*Médias seguidas pela mesma letra, na coluna, não diferem entre si, pelo teste de Scott-Knott a 5\% de probabilidade.

Entre as cultivares que sobrassaíram-se com maiores produtividades verificaram-se que a massa fresca do fruto variou entre $8,3 \mathrm{~kg}$ fruto $^{-1}$ (cultivar Opara $F_{1}$ ) e $11,0 \mathrm{~kg}$ fruto $^{-1}$ (cultivar Red Heaven) (Tabela 3). Resultados concordantes com Cardoso e Isaac (2010) que comentam ser os frutos comerciáveis entre 5,5 $\mathrm{kg}$ a $12,0 \mathrm{~kg}$, sendo em geral comercializados com peso médio entre $7,0 \mathrm{~kg}$ a $8,0 \mathrm{~kg}$. Oliveira et al. (2015) obtiverem massas frescas inferiores com $6,2 \mathrm{~kg}$ fruto $^{-1}$ (Cultivar Crimson Sweet) a $8,8 \mathrm{~kg}$ fruto $^{-1}$ (cultivar Denver). Valores estes próximos foram relatados por Carmo et al. (2015) que variação entre 7,1 a 9,0 kg fruto ${ }^{-1}$. Com massa fresca de fruto oscilando entre 8,3 a $12,3 \mathrm{~kg}$ fruto $^{-1}$, Silva et al. (2017) encontraram valores que superaram os observados no presente trabalho.

No que se refere ao número de frutos comerciais por planta e por hectare (Tabela 3) constataram-se variações entre 0,94 a 1,12 frutos planta $^{-1}$ e 5.246 e 6.234 frutos ha $^{-1}$, respectivamente; sem apresentar difrenças significativas entre as cultivares. Diferentes autores relatam valores entre 1,30 e 1,65 frutos planta $^{-1}$ (OLIVEIRA et al., 2015), 5.979 e 8.103 frutos hectare $^{-1}$ (CARMO et al., 2015) e 3.750 e 6.425 frutos hectare $^{-1}$ (SILVA et al., 2017).
Como pode ser observado o cultivo sob temperaturas mais amenas de inverno apresentaram menor rendimento médio com $38,9 \mathrm{t} \mathrm{ha}^{-1}$ comparativamente às temperaturas mais elevadas de verão, que alcançaram média de $51,7 \mathrm{t} \mathrm{ha}^{-1}$, o que vem confirmar a melhor adaptação da cultura a temperaturas mais elevadas (FILGUEIRA, 2008). Deve-se salientar ainda que as produtividades encontradas nos dois períodos de cultivo são bem superiores as produtividades mundiais relatadas pela de FAO (2018) de 33,6 t ha ${ }^{-1} \mathrm{e}$ nacional de 23,11 t ha ${ }^{-1}$ (IBGE, 2018).

\section{CONCLUSÕES}

Pelas suas características produtivas as cultivares de melancia Frevo, Red Heaven, Explorer e Ranger são as mais recomendadas para plantio sob condições de temperaturas mais amenas (inverno).

Sob condições de temperaturas mais elevadas (verão) as cultivares de melancia Opara $\mathrm{F}_{1}$, Ranger, Combat, Explorer, Conquista, Frevo e Red Heaven apresentam-se como as mais produtivas e ideais para cultivo no Submédio do Vale do São Francisco. 


\section{REFERÊNCIAS BIBLIOGRÁFICAS}

CARDOSO, M.C.; ISAAC, A.C. Cultivares de melancia recomendadas para o plantio em "terra firme" no Estado do Amazonas. Manaus: Embrapa Amazônia Ocidental, 2010. 5p. (Embrapa Amazônia Ocidental. Comunicado Técnico, 90).

CARMO, I.L.G.S.; SILVA, E.S.; MONTEIRO NETO, J.L.L.; TRASSATO, L.B.; MEDEIROS, R.D.; PORTO, D.S. Desempenho agronômico de cultivares de melancia no cerrado de Boa Vista, Roraima. RevistaAgro@mbiente On-Line, v. 9, n. 3, p. 268-274, 2015. http://dx.doi.org/10.18227/19828470ragro.v9i 3.2531 .

FAO. Agricultural production: primary crops. Disponível em: 〈http://www.fao.org>. Acesso em: 25 jun. 2018.

FERREIRA, D. F. Sisvar: a guide for its bootstrap procedures in multiple comparisons. Ciência e Agrotecnologia, v. 38, n. 2, p. 109112, 2014. http://dx.doi.org/10.1590/S141370542014000200001 .

FERREIRA, M.A.J.E.; QUEIROZ, M.A.; BRAZ, L.T.; VENCOVSKY, R. Correlações genótipicas, fenotípicas e de ambiente entre dez caracteres de melancia e suas implicações para o melhoramento genético. Horticultura Brasileira, v. 21, n. 3, p.438-444, 2003. http://dx.doi.org/10.1590/S010205362003000 300004.

FILGUEIRA, F.A.R. Novo manual de olericultura: Agrotecnologia moderna na produção e comercialização de hortaliças. Viçosa: Editora UFV, 2008. 402p.

IBGE - Instituto Brasileiro de Geografia e Estatística. Produção agrícola municipal. Disponível em: <www.sidra.ibge.gov.br>. Acesso em: 25 jun. 2018.

OLIVEIRA, J.B.; GRANGEIRO, L.C.; ESPINOLA SOBRINHO, J.; MOURA,
M.B.S.; CARVALHO, C.A.C. Rendimento e qualidade de frutos de melancia em diferentes épocas de plantio. Revista Caatinga, v. 28, n. 2, p. $19-25,2015$.

RESENDE, G.M.; COSTA, N.D.; DIAS; R.C.S. Clima. In: Sistema de produção de melancia, 6. 2010 a. Embrapa Semiàrido. Disponível em: $<$ https://sistemasdeproducao.cnptia.embrapa. br/Fontes HTML/Melancia/SistemaProducao Melancia/clima.htm>. Acesso em: 11 abr. 2018.

RESENDE, G.M.; COSTA, N.D.; DIAS; R.C.S. Plantio. In: Sistema de produção de melancia, 6. 2010b. Embrapa Semiàrido. Disponível em: $<$ https://sistemasdeproducao.cnptia. embrapa.br/FontesHTML/Melancia/SistemaPr oducaoMelancia/plantio.htm>. Acesso em: 11 abr. 2018.

Santos, H.G.; Jacomine, P.K.T.; Anjos, L.H.C.; Oliveira, V.A.; Lumbreras, J.F.; Coelho, M.R.; ALMEIDA, J.A.; ARAÚJO FILHO, J.C.; Oliveira, J.B.; Cunha, T.J.F. (Eds.) Sistema brasileiro de classificação de solos. 5. ed. Rio de Janeiro: Embrapa Solos, 2018. 356p.

SILVA, E.S.; CARMO, I.L.G.S.; MONTEIRO NETO, J.L.L.; MEDEIROS, R.D.; MENEZES, P.H.S.; RODRIGUEZ, C.A. Características agronômicas de cultivares de melancia nas condições do cerrado de Roraima, Brasil. Scientia Agropecuária, v. 3, n. $8, \quad$ p. $193-201, \quad 2017$. http://dx.doi.org/10.17268/sci.agropecu.2017. 03.02.

TEIXEIRA, A.H.C. Informações agrometeorológicas do Pólo Petrolina, PE/Juazeiro - 1963 a 2009. Petrolina: Embrapa Semiárido, 2010. 21p. (Embrapa Semiárido. Documentos, 233). 